\title{
Radiotherapy as a Treatment Option for Local Disease Control in Primary Cutaneous Diffuse Large B-Cell Lymphoma, Leg Type
}

\author{
Mara Zehnder ${ }^{a, c}$ Boyko Amarov ${ }^{b}$ André N. Abrunhosa-Branquinhod \\ Mirjana Maiwald-Urosevic ${ }^{g}$ Beda Mühleisen $^{c}$ leva Saulite $^{\mathrm{e}}$ \\ Florian Anzengruber ${ }^{a, g} \quad$ Laurence Imhof $^{a, g} \quad$ Alexander A. Navarini $^{c}$ \\ Antonio Cozzio $^{\mathrm{e}, \mathrm{g}}$ Reinhard Dummer ${ }^{\mathrm{a}, \mathrm{g}} \quad$ Florentia Dimitriou $^{\mathrm{a}, \mathrm{g}}$ \\ Emmanuella Guenova ${ }^{\mathrm{a}, \mathrm{f}, \mathrm{g}}$
}

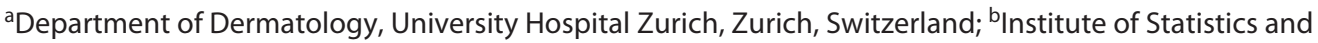
Econometrics, Sofia University "St. Kliment Ohridski", Faculty of Economics and Business Administration, Sofia, Bulgaria; 'Department of Dermatology, University of Basel, Basel, Switzerland; ' ${ }^{\mathrm{C}}$ Serviço de Radioterapia, Hospital Santa Maria - Centro Hospitalar Universitário Lisboa Norte (CHULN) E.P.E., Lisbon, Portugal; eDepartment of Dermatology, Kantonsspital St. Gallen, St. Gallen, Switzerland; 'Department of Dermatology, Lausanne University Hospital (CHUV) and Faculty of Biology and Medicine, University of Lausanne, Lausanne, Switzerland; 9Faculty of Medicine, University of Zurich, Zurich, Switzerland

\section{Keywords}

Radiotherapy · Chemotherapy · B-cell lymphoma · Primary cutaneous diffuse large B-cell lymphoma, leg type

\footnotetext{
Abstract

Background: Primary cutaneous diffuse large B-cell lymphoma, leg type (PCDLBCL, LT) is an aggressive lymphoma variant. Anthracycline-based chemotherapy with rituximab is recommended as first-line treatment. Radiotherapy (RT) has been considered as a therapeutic option for local disease control in patients with solitary or localized lesions. Methods: We report the results of a retrospective analysis of PCDLBC, LT patients treated either with RT alone or with physician's decision as first-line treatment, aiming to assess disease progression and/or first recurrence in these treatment groups. Results: We retrospectively analyzed 20 patients treated either with RT alone $(n=8)$ or with investigator's choice treatment $(n=12)$, which included chemotherapy
}

alone or combined with local therapy (RT and wide local excision). Complete response (CR) was achieved in 8 patients from the first group and 9 patients from the second group, with 1 treatment failure. Six patients treated with RT alone progressed with a median time to progression (TTP) of 12.5 months. In the second group, 5 patients progressed with a median TTP of 5.2 months. RT showed good local disease control in both groups without any skin relapses during the follow-up period. Conclusion: RT as first-line monotherapy followed by watchful waiting did not significantly improve the overall risk of disease progression but resulted in good local disease control. After progression, RT could still easily be combined with systemic treatment. The strength of this analysis needs to be evaluated in a larger patient cohort.

(C) 2022 The Author(s).

Published by S. Karger AG, Basel

Florentia Dimitriou and Emmanuella Guenova contributed equally as joint senior authors.
C) 2022 The Author(s).

Published by S. Karger AG, Basel

This is an Open Access article licensed under the Creative Commons Attribution-NonCommercial-4.0 International License (CC BY-NC) (http://www.karger.com/Services/OpenAccessLicense), applicable to the online version of the article only. Usage and distribution for commercial purposes requires written permission.
Correspondence to:

Emmanuella Guenova, emmanuella.guenova@unil.ch 


\section{Introduction}

Primary cutaneous B-cell lymphomas (PCBCL) include a heterogeneous group of mature lymphoproliferative disorders that account for approximately $25 \%$ of all primary cutaneous lymphomas (PCL) [1]. PCBCL comprise 3 major entities: primary cutaneous follicle-center lymphoma (PCFCL), primary cutaneous marginal zone lymphoma, and primary cutaneous diffuse large B-cell lymphoma, leg type (PCDLBCL, LT). PCFCL and primary cutaneous marginal zone lymphoma show an indolent, slowly progressive course with excellent prognosis, despite high recurrence rates [2]. By contrast, PCDLB$\mathrm{CL}, \mathrm{LT}$ represents a more aggressive lymphoma variant with unfavorable prognosis, frequent relapses, and often extracutaneous spreading [3]. Clinically, it is characterized by its predominant occurrence in elderly patients and presentation of solitary or multiple, rapidly growing, red to bluish-red firm tumors, preferentially on the lower legs $[1,4]$. Histologically, PCDLBCL, LT shows a high $(>70 \%)$ proliferation with medium to large cells with round nuclei, and immunophenotypically the neoplastic B cells are typically positive for CD20 and MUM-1 and show high bcl-2 expression, which explains the beneficial role of rituximab (anti-CD20 monoclonal antibody) in these patients [1].

Current studies have estimated the 5-year survival rate of PCDLBCL, LT to lie between 50 and 55\% [5-7]. Due to its aggressive course and its clinical behavior similar to systemic diffuse large B-cell lymphoma (DLBCL), an anthracycline-based chemotherapy including rituximab is recommended as first-line treatment for PCLBCL, LT. A combination of rituximab with cyclophosphamide, doxorubicin, vincristine, and prednisone ( $\mathrm{R}$ $\mathrm{CHOP}$ ) regimen is considered as a standard treatment option, whereas in elderly and/or fragile patients, this combination regimen has been replaced by less intensive combinations of rituximab and polychemotherapy $[8,9]$. Although radiotherapy (RT) is less effective than in other PCLBCL subtypes, it has been considered as a therapeutic option for local disease control in solitary or localized disease [1]. Recent studies suggest that chemotherapy, compared to stand-alone RT, remains the most effective single treatment with increased local control and improved survival; nevertheless, efficacy data of these treatment approaches are scarce $[10,11]$. Whether first-line RT in patients with localized disease leads to a significant improvement of survival is yet controversially discussed. Herein, we report the results of a singleinstitution, retrospective analysis of PCDLBC, LT pa- tients treated either with RT alone followed by watchful waiting or with physician's decision as first-line treatment, aiming to assess disease progression and/or recurrence in these treatment groups.

\section{Materials and Methods}

\section{Patients}

Medical records of 257 patients diagnosed with PCBCL in the Dermatology Clinic of the University Hospital of Zurich between December 1999 and June 2017 were retrospectively reviewed. Patients were followed every 4-12 weeks for at least 5 months after treatment initiation until October 2019 (closing date). Follow-up was determined on an individual basis according to the chosen treatment, with clinical examination and assessment every 4-12 weeks for the first year. In total, 20 patients diagnosed with PCDLBCL, LT and treated either with RT as first-line treatment followed by watchful waiting or with investigator's choice (chemotherapy alone or combined with RT, surgical excision) were identified. Treatment decision was made according to a interdisciplinary board discussion and was based on disease localization, extent of involved skin, lesion type, patient's performance status, and comorbidities. Diagnosis was based on local clinical and histological review; cases diagnosed before 2005 were re-classified according to the current WHO-EORTC (European Organization of Research and Treatment of Cancer) classification [12]. TNM staging was adopted according to the International Society for Cutaneous Lymphomas (ISCL) and the EORTC $[12,13]$. Demographic data including sex and age at first diagnosis, as well as comorbidities and multicomorbidities, defined as the presence of other conditions in addition to the index condition (PCDLBCL, LT) were assessed through their medical records. Comorbidity count was obtained from the number of individual comorbidities.

\section{Treatment Evaluation}

Disease characteristics, treatment, and outcome were retrospectively collected. Extent of involved skin was defined as solitary (T1a, T1b), multiple (T2a, T2b, T2c), or generalized (T3a, T3b) plaques and/or tumors [13]. In all patients, staging procedures were performed with standard methods, including physical examination, complete blood-cell count, computed tomography (CT) scan or positron emission tomography (PET)/CT scan, and bone marrow biopsy. Treatment response was evaluated according to clinical (e.g., dermoscopy) and radiological assessment as complete response (CR), partial response (PR), and progressive disease (PD). Objective response rate (ORR) was defined as CR and PR. Disease progression was further analyzed according to anatomic site and type as local (same site as primary tumor) and distant (different site of primary tumor) for cutaneous progression and as systemic for extracutaneous progression. Data analysis focused on disease outcome, including progression-free survival (PFS) rate. End points were assessed on the date of death or last patient contact by October 2019. Patients alive at the end of follow-up were included in the statistical analysis as censored observations. All analyses were conducted using statistical language $\mathrm{R}$ version 3.5. Reported $p$ values were accepted as statistically significant if less than 0.05 . 


\section{Results}

Patient Characteristics, Comorbidities, and Baseline Lactate Dehydrogenase

A total of 20 patients with PCDLBCL, LT and a median age of 79.5 years (range 53-95) at the time of diagnosis were included. Eleven patients were female and 9 were male. All patients had the clinical characteristics of a PCDLBCL, LT $[2,11,12]$. In 5 out of 20 patients (25\%), the disease at onset featured a solitary lesion (corresponding to T1) and in 15 of 20 patients (75\%) it included multiple tumors (corresponding to T2). The predilection site of disease at the time of diagnosis was the lower leg for most of the cases $(n=15)$. However, 5 patients presented with skin lesions on the trunk $(n=1)$, on the scalp $(n=$ 3 ), and on the upper extremities $(n=1)$ (Fig. 1). The 4 cases involving the trunk and scalp were re-evaluated according to the current classification. Due to clinical, histological, and immunophenotypical criteria, these patients are now recognized as PCDLBCL, LT [12]. Lesions' type included plaques $(n=2)$, tumors $(n=12)$, or both $(n=6)$. Three patients with tumor lesions and 1 patient with tumors and plaques presented with ulceration at the initial diagnosis. Extracutaneous disease with testicle involvement was diagnosed in 1 patient, which was subsequently treated with chemotherapy. Systemic involvement was not present in the rest of the patients $(n=19)$.

\section{Treatment Choice}

Radiotherapy (Group 1)

Eight out of 20 patients (40\%) without evidence of extracutaneous disease or disease outside of the radiation field were treated with local RT followed by watchful waiting as a first-line treatment (group 1). Seven patients were treated with curative intention; in 1 patient, RT was administered as palliative treatment due to advanced age. The RT group included 2 men and 6 women. The median age at diagnosis was 83 years (range 67-95). In this treatment group, 2 patients presented with solitary tumor lesions $(2 / 8)$ and 6 patients with multiple lesions $(6 / 8)$, either tumors $(n=4)$, or tumors and plaques $(n=2)$. Four out of 8 patients had an ulceration; 2 patients with solitary tumors, 1 with multiple tumors, and 1 with multiple tumors and plaques. The predominant site of involvement was the lower leg in all cases $(n=8)$.

RT was performed either as electron beam therapy $(n=2)$ or as superficial X-ray RT using Gulmay D3100 $\mathrm{X}$-ray unit (Gulmay GmbH, DE) $(n=6)$. RT treatment was chosen based on an interdisciplinary board discussion. RT plans and reports were performed according to

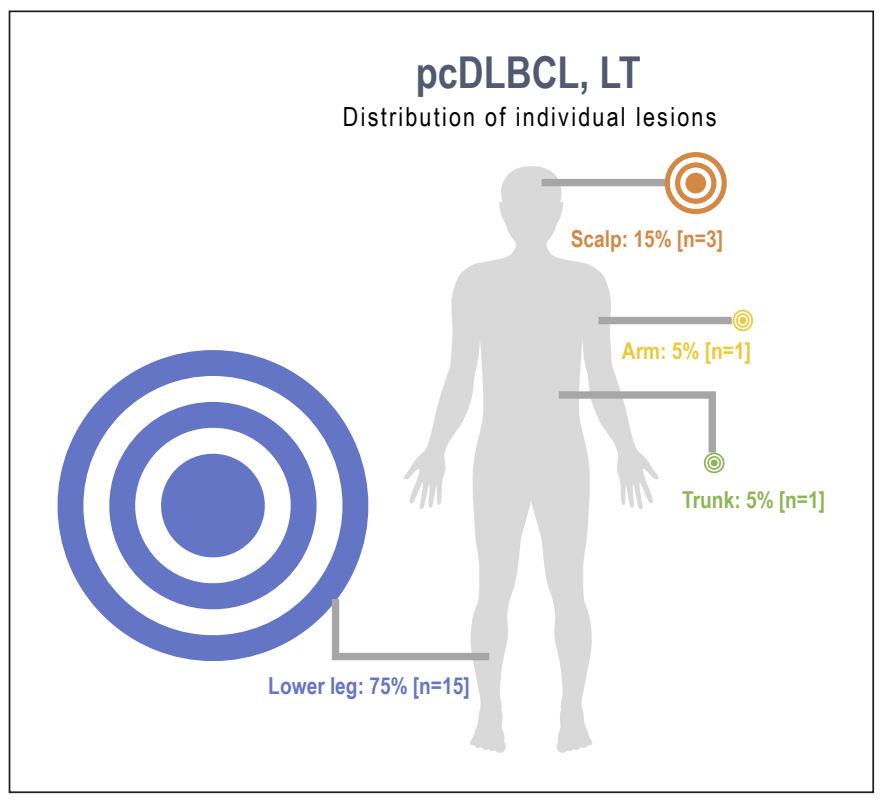

Fig. 1. Disease distribution at the time of diagnosis. The predilection site was the lower leg for most of the cases $(n=15)$. Five patients presented with skin lesions on the trunk $(n=1)$, on the scalp $(n=3)$, and on the upper extremities $(n=1)$.

the International Commission on Radiation Units (ICRU) standards. Patients' position was dependent on the tumor localization and, for each case, personalized devices were used to immobilize the patient for the treatment. Electron choice was according to physician's decision and was based on tumor thickness and localization. In patients with multiple lesions, all lesions were treated concurrently with multiple radiation fields. The median total dose applied was 34 Gy (range 16-40 Gy, voltage 40-100 kV), median fractionation size was 4 Gy per fraction (range 2-5 Gy/fraction). During the course of RT, all patients were treated as outpatients but were closely observed by hematological oncologists or dermatologists. Irradiation dose and treatment response are summarized in online supplementary Table S1 (for all online suppl. material, see www.karger.com/doi/10.1159/000522053). A margin of at least $1-2 \mathrm{~cm}$ of healthy skin was included in the radiation field according to the current guidelines. All patients were regularly evaluated after the end of RT to assess the clinical response and toxicities.

\section{Physician's Choice Treatment (Group 2)}

Twelve patients (60\%), 1 with systemic disease involvement, underwent a systemic or local treatment other than RT alone according to physician's decision (group 2). This patient group included 5 female and 7 male pa- 
Table 1. Disease progression and outcome

\begin{tabular}{|c|c|c|c|c|c|c|}
\hline & \multicolumn{3}{|c|}{ RT group } & \multicolumn{3}{|c|}{ ID group } \\
\hline Subjects, $n$ & \multicolumn{3}{|l|}{8} & \multicolumn{3}{|l|}{12} \\
\hline Disease relapse or progression, $n$ & \multicolumn{3}{|c|}{6} & \multicolumn{3}{|c|}{5} \\
\hline TTP, median (range), months & \multicolumn{3}{|c|}{$12.5(6-31.2)$} & \multicolumn{3}{|c|}{$5.2(5.1-6.3)$} \\
\hline \multicolumn{7}{|l|}{ Site of progression } \\
\hline Local & \multicolumn{3}{|l|}{3} & \multicolumn{3}{|l|}{2} \\
\hline Distant & \multicolumn{3}{|l|}{1} & \multicolumn{3}{|l|}{2} \\
\hline Systemic & \multicolumn{3}{|l|}{2} & \multicolumn{3}{|l|}{1} \\
\hline \multicolumn{7}{|l|}{ Subsequent treatment } \\
\hline \multicolumn{7}{|l|}{ Skin-directed only } \\
\hline RT & \multicolumn{3}{|l|}{2} & \multicolumn{3}{|l|}{2} \\
\hline Wide local excision & \multicolumn{3}{|l|}{1} & \multicolumn{3}{|l|}{-} \\
\hline \multirow[t]{2}{*}{ Systemic treatment ( \pm skin-directed treatment) } & \multicolumn{3}{|l|}{3} & \multicolumn{3}{|l|}{3} \\
\hline & $\mathrm{CR}$ & PR & PD & CR & PR & PD \\
\hline \multicolumn{7}{|l|}{$\begin{array}{l}\text { Response to subsequent treatment } \\
\text { Skin-directed only }\end{array}$} \\
\hline $\mathrm{RT}$ & 2 & - & - & 1 & - & 1 \\
\hline Wide local excision & - & - & 1 & - & - & - \\
\hline Systemic treatment ( \pm skin-directed treatment) & - & 2 & 1 & 1 & 1 & 1 \\
\hline \multicolumn{7}{|l|}{ Status } \\
\hline Alive & \multicolumn{3}{|l|}{3} & \multicolumn{3}{|l|}{5} \\
\hline Dead & \multicolumn{3}{|l|}{1} & \multicolumn{3}{|l|}{2} \\
\hline Lost to FU & \multicolumn{3}{|l|}{4} & \multicolumn{3}{|l|}{5} \\
\hline
\end{tabular}

$\mathrm{RT}$, radiotherapy; ID, investigator's decision; $C R$, complete response; $P R$, partial response; PD, progressive disease; TTP, time to progression; FU, follow-up.

tients with a median age of 69 years (range 47-94 years). Investigator's decision treatment included combination systemic treatment with RT in 7 out of 12 patients. RT was combined with R-CHOP in 1 patient, $\mathrm{CHOP}$, doxorubicin, and rituximab alone (anti-CD20 monoclonal antibody) either administered intralesionally $(n=3)$ or systemically $(n=1)$. Other treatments included operation alone in 3 patients with solitary lesions on the scalp, on the trunk, and on the upper extremities, as well as RDHAP (rituximab, dexamethasone, cytarabine, cisplatin) in 1 patient with systemic involvement, previously treated with R-CHOP. Six patients presented with tumor lesions (solitary [2/6], multiple [3/6], or generalized [1/6]), 1 patient with a solitary plaque, and 4 patients with multiple tumors and plaques. No ulcerative lesions were observed.

\section{Treatment Outcome}

ORR was achieved in 19 out of 20 patients; 8 from group 1 and 11 from group 2 (Table 1 ). One patient (1/19) treated with RT in combination with doxorubicin from the second treatment group did not respond to the initial treatment (PD). At the end of the observational period, 5 out of 19 patients without extracutaneous disease at the time of diagnosis eventually developed a systemic involvement. As of October 2019 and after a median followup time of 30.9 months (2.8-147 months), 8 patients were alive and 3 had deceased, 1 due to lymphoma and 2 due to medical condition(s) other than lymphoma, including reduced performance status. Nine patients were lost to follow-up, though after a median follow-up time of 36.8 months (2.8-147 months).

\section{Radiotherapy (Group 1)}

In the RT-alone group, 6 out of 8 patients eventually relapsed. Disease relapse characteristics are summarized in Table 1 . Three patients (3/6) had a local relapse and were subsequently treated with skin-directed treatment, including RT or wide local excision, with subsequent CR in 2 patients and $\mathrm{PD}$ in 1 patient. One patient (1/6) was diagnosed with a distant relapse and 2 patients (2/6) with systemic relapse prompting a systemic treatment with rituximabbased chemotherapy, either with R-CHOP for the patients 
Table 2. Baseline characteristics, response, and comorbidities compared between the two groups

\begin{tabular}{|c|c|c|c|c|}
\hline Characteristic & $N$ & $\mathrm{RT}(n=8)^{1}$ & ID $(n=12)^{1}$ & $p$ value $^{2}$ \\
\hline Gender, $n(\%)$ & 20 & & & 0.2 \\
\hline Female & & $6(75)$ & $5(42)$ & \\
\hline Male & & $2(25)$ & $7(58)$ & \\
\hline Age at first diagnosis, years & 20 & $83(78,88)$ & $69(59,85)$ & 0.10 \\
\hline Clinical localization, $n(\%)$ & 20 & & & 0.3 \\
\hline Lower leg & & $8(100)$ & $7(58)$ & \\
\hline Scalp & & $0(0)$ & $3(25)$ & \\
\hline Trunk & & $0(0)$ & $1(8.3)$ & \\
\hline Upper extremities & & $0(0)$ & $1(8.3)$ & \\
\hline Lesion type, $n$ (\%) & 20 & & & 0.5 \\
\hline Both & & $2(25)$ & $4(33)$ & \\
\hline Plaque & & $0(0)$ & $2(17)$ & \\
\hline Tumor & & $6(75)$ & $6(50)$ & \\
\hline Ulceration, $n(\%)$ & 20 & & & 0.014 \\
\hline Nonulcerated & & $4(50)$ & $12(100)$ & \\
\hline Ulcerated & & $4(50)$ & $0(0)$ & \\
\hline Response, $n(\%)$ & 20 & & & 0.5 \\
\hline $\mathrm{CR}$ & & $8(100)$ & $9(75)$ & \\
\hline PD & & $0(0)$ & $1(8.3)$ & \\
\hline PR & & $0(0)$ & $2(17)$ & \\
\hline $\mathrm{LDH}, n(\%)$ & 18 & & & 0.6 \\
\hline$<$ ULN & & $6(75)$ & $9(90)$ & \\
\hline$>$ ULN & & $2(25)$ & $1(10)$ & \\
\hline Unknown & & 0 & 2 & \\
\hline Comorbidities, $n$ (\%) & 20 & & & 0.4 \\
\hline 0 & & $1(12)$ & $3(25)$ & \\
\hline 1 & & $2(25)$ & $1(8.3)$ & \\
\hline 2 & & $3(38)$ & $5(42)$ & \\
\hline 3 & & $2(25)$ & $0(0)$ & \\
\hline 4 & & $0(0)$ & $1(8.3)$ & \\
\hline 5 & & $0(0)$ & $2(17)$ & \\
\hline
\end{tabular}

Comorbidity conditions were chosen on the basis that they require a long-term pharmacological therapy. For each patient, the number of comorbidities was summed to obtain a comorbidity count. The following comorbidities were identified: cardiovascular (valvular, coronary, and hypertensive heart disease), respiratory, renal (chronic and acute renal insufficiency), musculoskeletal, metabolic, hematological, oncologic (melanoma, breast and prostate cancer), and psychiatric (depression). RT, radiotherapy; ID, investigator's decision; $\mathrm{CR}$, complete response; $\mathrm{PR}$, partial response; $\mathrm{PD}$, progressive disease; $\mathrm{LDH}$, lactate dehydrogenase; ULN, upper limit of normal. ${ }^{1}$ Statistics presented: $n$ (\%); median (IQR). ${ }^{2}$ Statistical tests performed: Fisher's exact test; Wilcoxon rank-sum test. with systemic and distant relapse $(n=2)$ or with MATRix regimen (methotrexate, cytarabine, thiotepa, rituximab; $n=1$ ) for the patient with systemic relapse, resulting in PR and PD, respectively. Notably, despite local relapse on the same site as the primary tumor in 3 patients, disease relapse was not observed on previously irradiated areas within the RT field. Median TTP was 12.5 months (range 6-31.2 months). Only 2 patients (25\%) from the RT-only group showed a sustained CR after local treatment.

\section{Physician's Choice Treatment (Group 2)}

In the second group, CR rates were achieved in $9 / 12$ patients, with treatment failure (PD) in 1 of 12 patients treated with RT in combination with doxorubicin due to advanced age. Five patients (41.7\%) from this treatment group showed a sustained CR and 5 patients eventually relapsed after a median TTP of 5.2 months (range 5.1-6.3 months) (Table 1). Two patients diagnosed with local relapse were subsequently treated with RT alone. RT alone $(n=2)$ for local relapse or RT combined with R-RCHOP $(n=1)$, intralesional rituximab $(n=1)$ and ESHAP (etoposide, solu-medrol, cytarabine, cisplatin; $n=1$ ) for dis- 


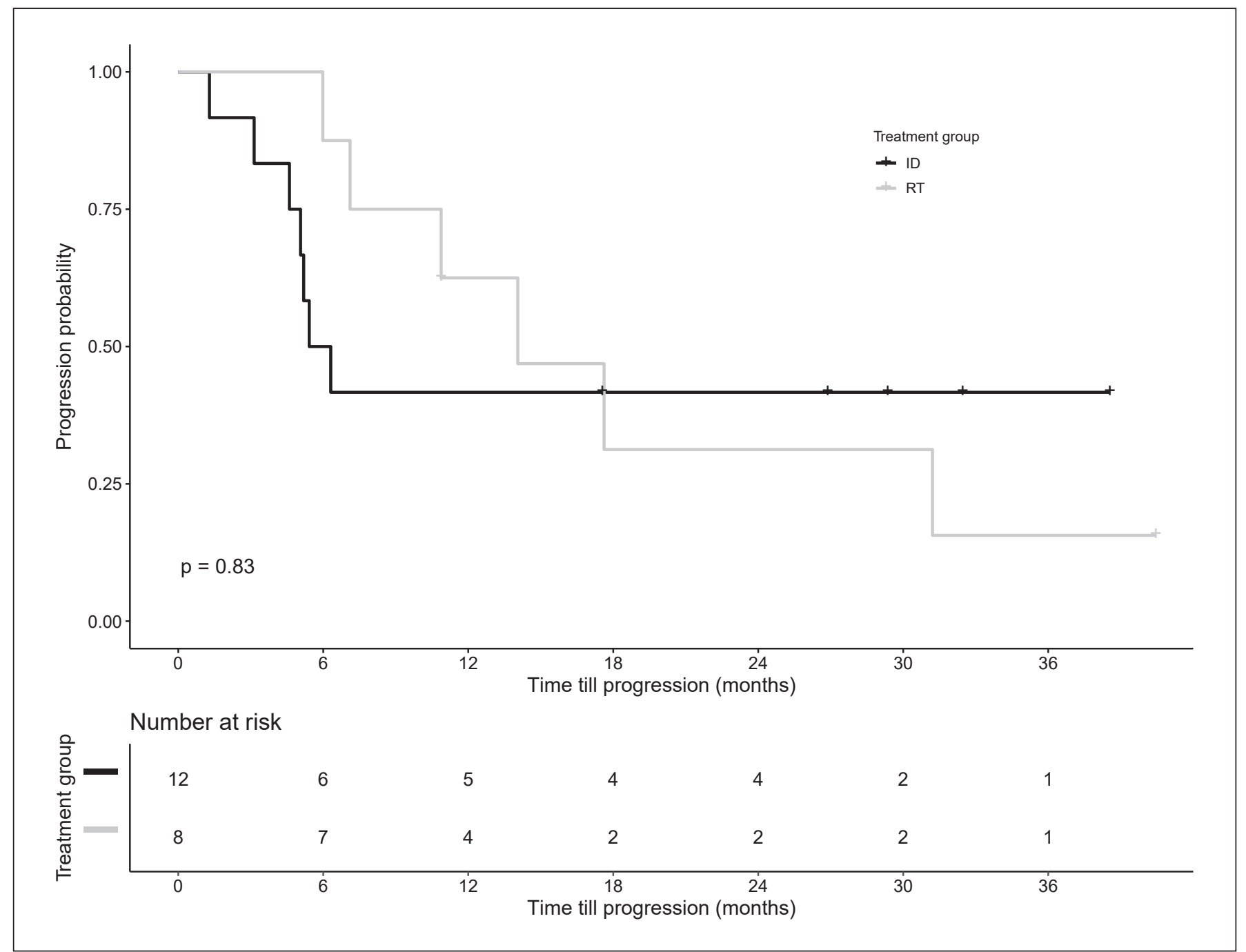

Fig. 2. Probability of progression-free survival in both treatment groups (comparing RT alone vs. ID as first-line treatment). RT, radiotherapy; ID, investigator's decision.

tant $(n=2)$ or systemic $(n=1)$ relapse were the treatments that were preferred upon disease relapse in this treatment group. In this group, RT alone showed high rates of local disease control, as no skin relapses on previously irradiated areas within the radiation field were observed.

\section{Impact of Patients' Characteristics, Disease}

Characteristics, and Baseline Lactate Dehydrogenase on Treatment Decision

Patients' characteristics, including gender and age, disease characteristics, including clinical localization, lesion type, and ulceration, as well as baseline lactate dehydrogenase and treatment response were analyzed and compared within the two groups (Table 2) in order to assess the impact of these characteristics on the firstline treatment decision. Patients' comorbidities were defined as described on the Materials and Methods section. The following comorbidities were identified: cardiovascular (valvular, coronary, and hypertensive heart disease), respiratory, renal (chronic and acute renal insufficiency), musculoskeletal, metabolic, hematological, oncologic (melanoma, breast and prostate cancer), and psychiatric (depression). Regarding the abovementioned comorbidities, no statistically significant differences were identified between the two groups. Similarly, no statistically significant differences for the above-mentioned baseline characteristics were identi- 


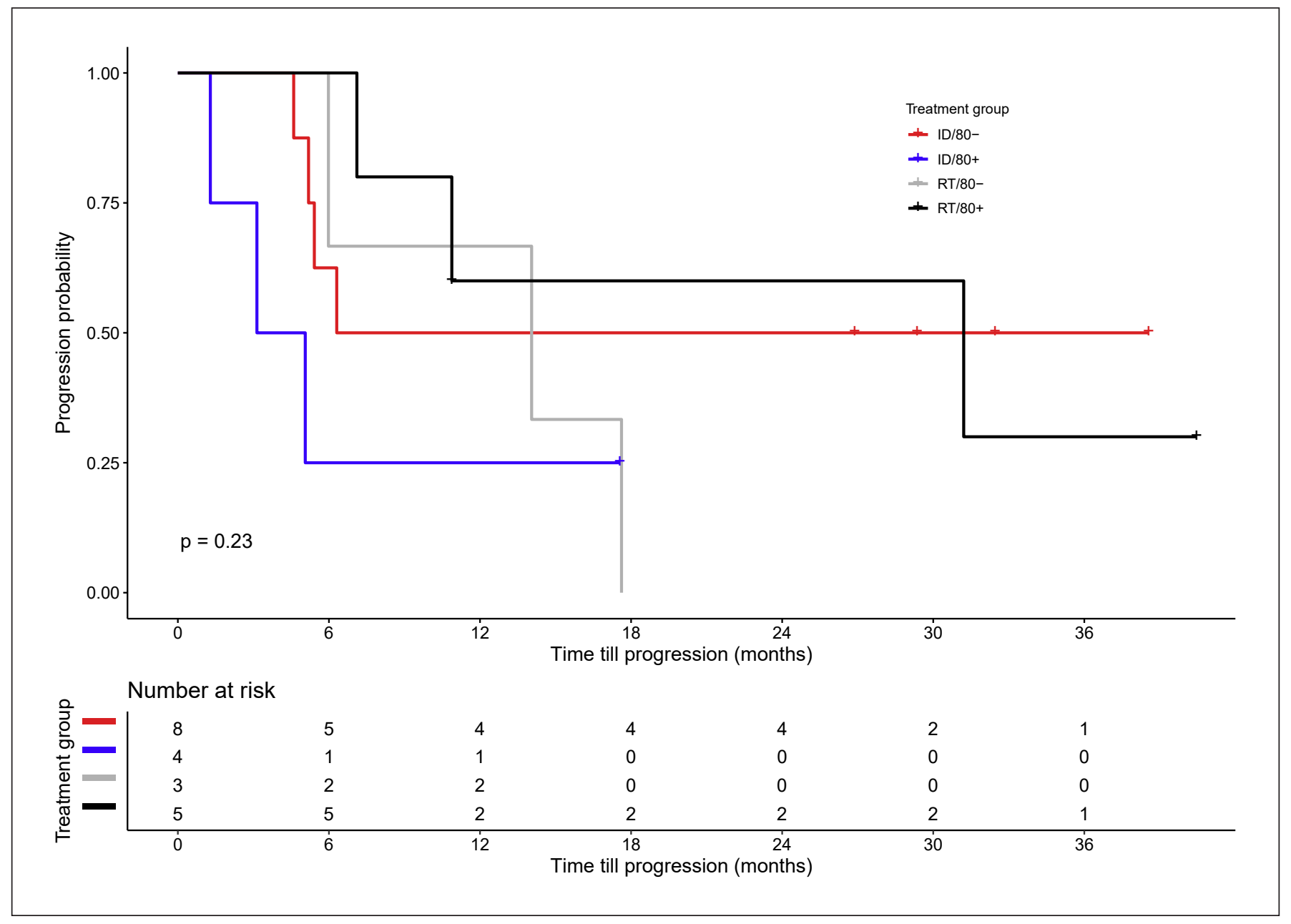

Fig. 3. Probability of progression-free survival according to age in both treatment groups (cutoff 80 years). RT, radiotherapy; ID, investigator's decision.

fied, although first-line RT was preferred in patients with local ulceration $(p=0.014)$.

\section{Disease Progression and Survival Probability}

At the end of the median follow-up time, PFS was 55\% and $50 \%$ at 6 and 12 months, respectively, whereas at the end of the median follow-up period (30 months), PFS was $35 \%$. We further compared the two treatment groups according to disease progression risk (Fig. 2), where no benefit of RT or investigator's decision was found for PFS $(p=0.83)$. Similarly, a comparison between RT and investigator's decision according to age (cutoff 80 years, Fig. 3) and extension of disease (Fig. 4) showed no significant difference in disease progression between the two groups ( $p=0.23$ and 0.31 , respectively).

\section{Discussion}

PCDLBCL, LT represents an indolent CBCL characterized by an aggressive course with a 5 -year disease-specific survival of approximately $50 \%$ [1]. Treatment recommendations, based on the EORTC/ISCL recommendations [14] and German S2k guidelines [15], indicate that PCDLBCL, LT should be treated with an anthracycline-based chemotherapy based on rituximab. The role of RT for the management of local disease has been controversially discussed. In general, PCBCL is considered to be radiosensitive; RT in PCFCL and PCMCL results in excellent local control rates in patients with $\mathrm{T} 1$ or $\mathrm{T} 2$ disease, without extracutaneous spread [16]. On the contrary, PCDLBCL, LT often leads to relapses and develops extracutaneous disease $[17,18]$. RT has been previously 


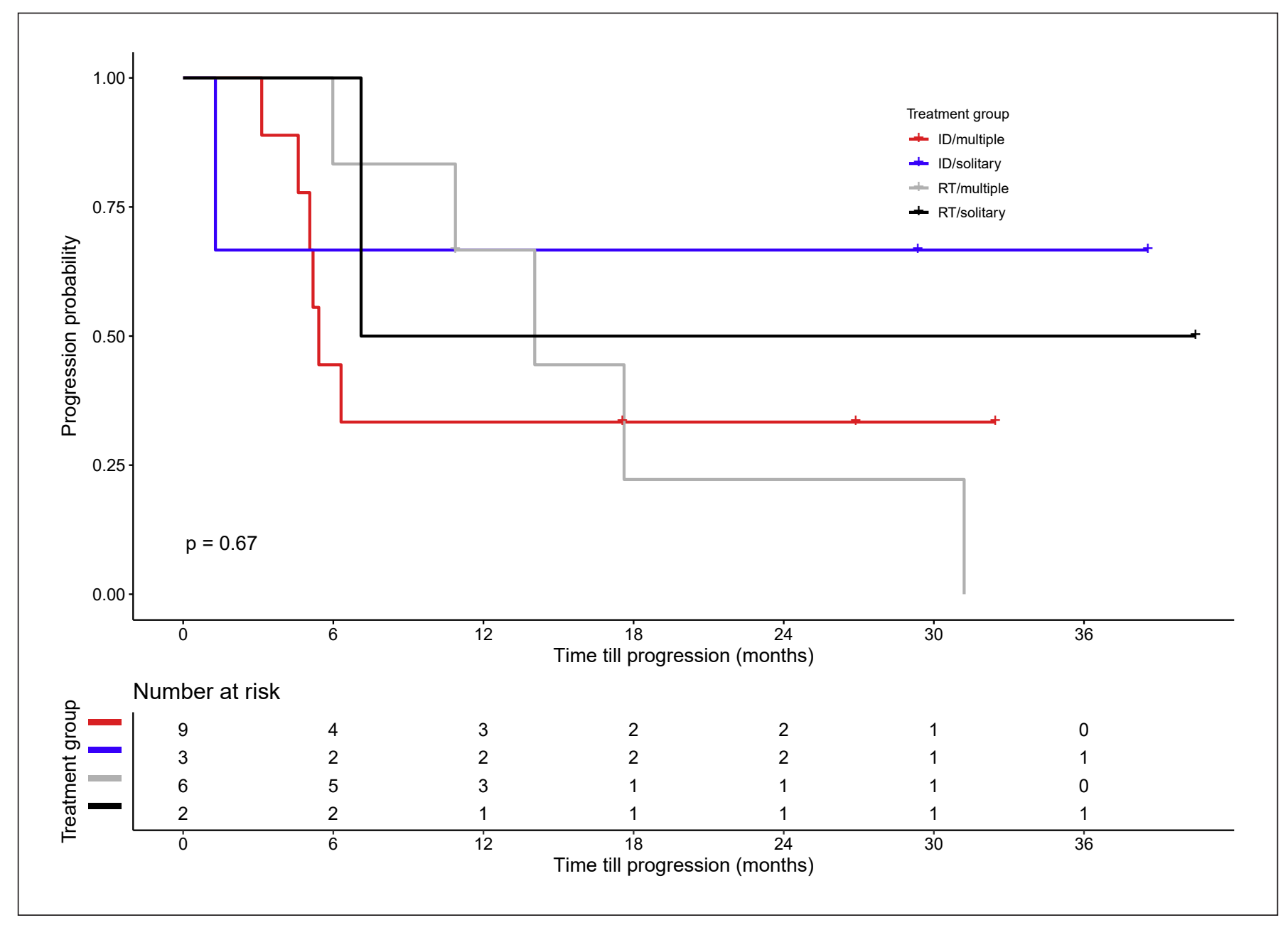

Fig. 4. Probability of progression-free survival according to extension of disease in both treatment groups. Extension of disease is described as solitary (T1a, T1b), multiple (T2a, T2b, T2c), or generalized (T3a, T3b). RT, radiotherapy; ID, investigator's decision.

examined as a skin-directed treatment option for local disease control, though without evidence of significant improvement on distant and systemic disease relapse rates.

In this study, we evaluated the treatment outcome of 20 patients diagnosed with PCDLBCL, LT, of which 8 were treated with RT alone as a first-line treatment and 12 with investigator's choice. Although the two groups showed some noticeable differences in patients' characteristics and extent of disease, which can influence the comparability of the groups, it seems that local RT may be a treatment option for local disease control in patients with PCDLBCL, LT without extracutaneous spread or disease outside of the radiation field. Previous studies reported that RT in PCDLBCL, LT led to CR in $88 \%$ of the patients, of which 58\% relapsed and 30\% developped extracutaneous progression [14]. Similarly, in a multicenter, retrospective analysis, ORR with local RT in patients with PCDLBCL, LT was $93 \%$ CR, but 7 patients (7/25) relapsed and $11 / 25$ died due to lymphoma [18]. In the current patient cohort, RT alone initially resulted in CR in all patients, regardless of lesion type and extent of involved skin. However, patients in this treatment group either had solitary or multiple lesions, localized on the same predilection type (lower leg) and, thus, accessible by local RT, which underlines that RT alone was preferred by the investigators for specific lesion types and disease localizations. Since the vast majority of the patients relapsed (75\%), the relapse rates in this patient cohort are higher than those previously reported, though a direct compari- 
son with these data could be misleading. In previous reports, overall survival and PFS after 5-year follow-up in patients treated with RT were calculated at $40 \%$ and 59\%, respectively, without any difference between solitary and multiple lesions [18]. In this study, the PFS rate at the median time of observation (30 months) was $30 \%$ for the RT-alone group and 35\% for the whole patient cohort. Notably, no statistically significant difference was found in the disease progression distribution between RT alone and investigator's decision, even if compared according to age (cutoff 80 years) and extension of disease. However, it is important to note that gender and age were unbalanced between the groups. There was a female predominance in the investigator's choice group and a male predominance in the RT-alone group, which can influence the comparability between the two groups.

Although our data did not reveal any systematic differences in the disease progression distribution, median TTP differed between the two groups in favor of RT alone (12.5 vs. 5.2 months). Eventually, a significant proportion of the patients from both treatment groups progressed and systemic treatment with chemotherapy was applied in the majority of the patients. These considerable relapse rates are in accordance with those observed in previous studies, which underlines that disease relapse should not be neglected, despite initial favorable response [18]. Although RT as a first-line monotherapy did not improve the risk of disease progression, it significantly improved the local disease control in both groups, since no skin relapses on previously irradiated areas were observed during the follow-up period of the study. These results, together with previous studies, could support the use of RT as salvage treatment in these patients, especially in the presence of important comorbidities that prevent the use of aggressive chemotherapy agents, such as secondary malignancies and hematological diseases. To date, several studies suggest systemic treatment with less intensive regimens in elderly patients with diminished general condition and organ function. In real life, R-CHOP has been largely replaced with rituximab, combined with polychemotherapy or lower doses of anthracycline (R-mini$\mathrm{CHOP}$ ) in these patients, without any negative impact on disease outcome [19]. Those dose adjustments or reduced schemes for systemic treatment can be ideally combined with RT as a salvage treatment for localized disease. Although our study did not examine the dose efficacy, Specht et al. [16] recommends 36-40 Gy with 40 Gy in patients without any systemic treatment and safety margins of 1-2 cm. Most lesions can be treated effectively with electrons, usually 6-9 MeV.

Radiotherapy in PCDLBCL, LT
The main strength of our study is the detailed clinical information available for the PCDLBCL, LT patients. Despite the long median follow-up time of 30.9 months, 9 patients were eventually lost to follow-up, which is a limitation of the analysis. The small number of patients, the comparability of the two groups, the variability in technologies, and variety of therapies through this long treatment period and the retrospective nature of the data are further limitations. Furthermore, patients treated with RT had less extensive disease than those treated with systemic treatment. However, given the rarity of PCDLBCL, LT, our study provides interesting conclusions on the effect of local RT for the management of local disease in this cohort.

\section{Conclusion}

According to this retrospective study, we conclude that first-line RT followed by watchful waiting compared to investigator's choice systemic treatment did not significantly improve the overall risk of disease progression in PCDLBCL, LT. RT as a first-line treatment for specific lesion types and disease localization resulted in local disease control, and even upon disease relapse, it could still be easily combined with systemic treatment in PCDLBCL, LT. Taking into consideration the limited therapeutic spectrum available, these data suggest that RT may be a valuable treatment option in patients with PCDLBCL, LT, especially in the elderly and/or in the presence of important comorbidities that prevent the use of systemic chemotherapy agents.

\section{Key Message}

RT as first-line monotherapy did not improve the overall risk of progression in PCDLBCL, LT.

\section{Statement of Ethics}

Written informed consent for retrospective analysis of PCBCL patients was approved by local ethics committee (KEK-ZH 20140193). All subjects have given their written informed consent for this study.

\section{Conflict of Interest Statement}

F.A. has received honoraria from AbbVie, Celgene, Leo Pharma, Galderma, Janssen-Cilag, Almirall, Eli Lilly, and Novartis, but has no financial interest, nor holds any shares of any pharmaceuti-

Dermatology 2022;238:967-976

DOI: $10.1159 / 000522053$ 
cal company. R.D. has intermittent, project-focused consulting and/or advisory relationships with Novartis, Merck Sharp \& Dohme, Bristol-Myers Squibb, Roche, Amgen, Takeda, Pierre Fabre, Sun Pharma, and Sanofi outside the submitted work. E.G. has intermittent, project-focused consulting and/or advisory relationships with Mallinckrodt, Takeda, Helsinn, and Novartis, outside the submitted work. F.D., M.Z., M.M.-U., B.A., A.N.A.-B., I.S., L.I., A.C., and A.A.N. have no conflicts of interest to declare.

\section{Funding Sources}

This project was supported by the Jubiläumsstiftung von SwissLife, the Promedica Stiftung (1406/M and 1412/M), a further anonymous Foundation, the Swiss Cancer Research Foundation (KFS-4243-08-2017), the Clinical Research Priority Program (CRPP) of the University of Zurich, the Swiss National Science Foundation (PMPDP3_151326), and the European Academy of Dermatology and Venereology (PPRC-2019-20). The funders had no role in study design, data collection and analysis, decision to publish, or preparation of the manuscript.

\section{Author Contributions}

Study concepts: M.Z., E.G. Study design: M.Z., E.G. Data acquisition: M.Z., M.M.-U., B.M., F.D., E.G. Quality control of data and algorithms: F.D., E.G. Data analysis and interpretation: M.Z., F.D., B.A. Statistical analysis: F.D., B.A. Manuscript preparation: M.Z., F.D. Manuscript editing: F.D., E.G. Manuscript review: B.A., A.N.A.-B., M.M.-U., I.S., F.A., L.I., A.A.N., A.C., R.D., E.G.

\section{Data Availability Statement}

All data generated or analyzed during this study are included in this article. Further enquiries can be directed to the corresponding author.

\section{References}

1 Willemze R, Jaffe ES, Burg G, Cerroni L, Berti E, Swerdlow SH, et al. WHO-EORTC classification for cutaneous lymphomas. Blood. 2005;105(10):3768-85

2 Willemze R. Primary cutaneous B-cell lymphoma: classification and treatment. Curr Opin Oncol. 2006;18(5):425-31.

3 Graham PM, Richardson AS, Schapiro BL, Saunders MD, Stewart DM. Spontaneous regression of primary cutaneous diffuse large Bcell lymphoma, leg type with significant T-cell immune response. JAAD Case Rep. 2018; 4(4):305-9.

4 Selva R, Violetti SA, Delfino C, Grandi V, Cicchelli S, Tomasini C, et al. A literature revision in primary cutaneous B-cell lymphoma. Indian J Dermatol. 2017;62(2):146-57.

5 Suarez AL, Querfeld C, Horwitz S, Pulitzer M, Moskowitz A, Myskowski PL. Primary cutaneous B-cell lymphomas: part II. Therapy and future directions. J Am Acad Dermatol. 2013; 69(3):343.e1-11; quiz 355-6.

6 Vermeer MH, Geelen FA, van Haselen CW, van Voorst Vader PC, Geerts ML, van Vloten WA, et al. Primary cutaneous large B-cell lymphomas of the legs. A distinct type of $\mathrm{cu}^{-}$ taneous B-cell lymphoma with an intermediate prognosis. Dutch Cutaneous Lymphoma Working Group. Arch Dermatol. 1996 132(11):1304-8.

7 Koens L, Vermeer MH, Willemze R, Jansen PM. IgM expression on paraffin sections distinguishes primary cutaneous large B-cell lymphoma, leg type from primary cutaneous follicle center lymphoma. Am J Surg Pathol. 2010;34(7):1043-8.

8 Coiffier B, Lepage E, Briere J, Herbrecht R, Tilly H, Bouabdallah R, et al. CHOP chemo- therapy plus rituximab compared with $\mathrm{CHOP}$ alone in elderly patients with diffuse large-Bcell lymphoma. N Engl J Med. 2002;346(4): 235-42.

9 Coiffier B, Thieblemont C, Van Den Neste E, Lepeu G, Plantier I, Castaigne S, et al. Longterm outcome of patients in the LNH-98.5 trial, the first randomized study comparing rituximab-CHOP to standard CHOP chemotherapy in DLBCL patients: a study by the Groupe d'Etudes des Lymphomes de l'Adulte. Blood. 2010;116(12):2040-5.

10 Hamilton SN, Wai ES, Tan K, Alexander C, Gascoyne RD, Connors JM. Treatment and outcomes in patients with primary cutaneous B-cell lymphoma: the BC Cancer Agency experience. Int J Radiat Oncol Biol Phys. 2013; 87(4):719-25.

11 Chen ST, Barnes J, Duncan L. Primary cutaneous B-cell lymphomas - clinical and histopathologic features, differential diagnosis, and treatment. Semin Cutan Med Surg. 2018; 37(1):49-55.

12 Willemze R, Cerroni L, Kempf W, Berti E, Facchetti F, Swerdlow SH, et al. The 2018 update of the WHO-EORTC classification for primary cutaneous lymphomas. Blood. 2019; 133(16):1703-14.

13 Kim YH, Willemze R, Pimpinelli N, Whittaker S, Olsen EA, Ranki A, et al. TNM classification system for primary cutaneous lymphomas other than mycosis fungoides and Sezary syndrome: a proposal of the International Society for Cutaneous Lymphomas (ISCL) and the Cutaneous Lymphoma Task Force of the European Organization of Research and Treatment of Cancer (EORTC). Blood. 2007; 110(2):479-84.
14 Senff NJ, Noordijk EM, Kim YH, Bagot M, Berti E, Cerroni L, et al. European Organization for Research and Treatment of Cancer and International Society for Cutaneous Lymphoma consensus recommendations for the management of cutaneous B-cell lymphomas. Blood. 2008;112(5):1600-9.

15 Dippel E, Assaf C, Becker JC, von BergweltBaildon M, Beyer M, Cozzio A, et al. S2k Guidelines - Cutaneous Lymphomas Update 2016 - Part 2: treatment and follow-up (ICD10 C82-C86). J Dtsch Dermatol Ges. 2018;16(1):112-22.

16 Specht L, Dabaja B, Illidge T, Wilson LD, Hoppe RT; International Lymphoma Radiation Oncology Group. Modern radiation therapy for primary cutaneous lymphomas: field and dose guidelines from the International Lymphoma Radiation Oncology Group. Int J Radiat Oncol Biol Phys. 2015; 92(1):32-9.

17 Ju R, Toonstra J, Meijer OW, Noordijk EM, Willemze R. Treatment of primary cutaneous B-cell lymphomas of follicle center cell origin: a clinical follow-up study of 55 patients treated with radiotherapy or polychemotherapy. J Clin Oncol. 1996;14(2):549-55.

18 Senff NJ, Hoefnagel JJ, Neelis KJ, Vermeer MH, Noordijk EM, Willemze R, et al. Results of radiotherapy in 153 primary cutaneous BCell lymphomas classified according to the WHO-EORTC classification. Arch Dermatol. 2007;143(12):1520-6.

19 Grange F, Joly P, Barbe C, Bagot M, Dalle S, Ingen-Housz-Oro $S$, et al. Improvement of survival in patients with primary cutaneous diffuse large B-cell lymphoma, leg type, in France. JAMA Dermatol. 2014;150(5):535-41. 